$621.914 .2 .014: 539.377 .096$

\title{
Thermal Cracks of Carbide Face Milling Cutter*
}

\author{
By Noboru SHINOZAKI**
}

Under severe cutting conditions of carbide face-milling operation, cracks first come out almost perpendiculary to the cutting edge on both rake and relief surface of blades, and next, cracks parallel to the cutting edge appear according to circumstances. These cracks have negative effects on the durability of a cutter and especially when cutting fluids are applied, they occur remarkably and the cutter is damaged at an early stage.

Situation and circumstances under which cracks occur have three characteristic features. (1) Cracks occur easily when the rate of cooling is better or the cutting condition is severe, namely the effect of thermal stress which depends on temperature difference of cutter surface is large. (2) Occurrence of cracks has size effect. (3) Cracks occur as a saturation curve.

It is reported that probability $P_{V}$ of cracks occurring by one thermal tensile stress $\sigma_{T}$ is given by the following equation and the features of crack occurrence are explained qualitatively.

$$
\begin{aligned}
P_{V}=1 & -\exp \left[-\frac{r_{m} L d \sec c}{K(m+1)}\left(\frac{\sigma_{T}}{\sigma_{0}}\right)^{m}\right. \\
2 r_{m} & : \text { thickness of carbide tip } \\
L d \sec c & : \text { size of chip contact area } \\
\sigma_{0} & : \text { strength const. of tip } \\
K & : \text { const. depending on relative heat transfer coeff. } \\
m & : \text { material const. }
\end{aligned}
$$

\section{Introduction}

It is generally known that the tool life of a Carbide Face Milling cutter is shorter than that of a lathe tool, (this phenomenon used to be chiefly attributed to damages on the cutting edge brought about by discontinuous cutting), and for this reason, the carbide face milling cutter had been applied little to steel workpieces until some years ago. But, with the improvements of the qualities of carbide face tools, such damages have come to be scarce, and thus, carbide milling became applicable to considerable severe cutting operations. Nowadays, everybody knows that the face milling operation is introduced into the fields of shapering and planing. Practically speaking, face milling operation consists of "cutting" (heated) and "free rotation in the air" (cooled) in each rotation of the cutter; hence, it was commonly believed in the former days that milling operation was preferable to lathe operation from the viewpoint of tool life, because the cutting

* Received 26 th November, 1960.

** Research Fellow, The Institutes of Physical and Chemical Research, Bunkyo-ku, Tokyo. edge is more effectively cooled than in the continuous cutting. Meanwhile carbide or ceramic tools, which are particulary weak under thermal stress, appeared as new tool materials and the cutting condition became severer, thereupon, the characteristic repetitions of "heating" and "cooling" came to exert, on the contrary, worse effect on tool life. Researches on the fatigue fracture phenomenon by Opitz and Frölich ${ }^{(1)}$, and Okushima and $\mathrm{Hoshi}^{(2)}$ may well be referred to in this respect.

Application of cutting fluids in this respect will cool the cutting edge during the free rotation in the air to such an extent that the resulted temperature difference on tool surface may cause damage to the tool at the early period of run. This may easily be realized by our experience that application of cutting fluid to the face milling cutter operated under considerably severe cutting conditions is likely to shorten the tool life. At the same time, Roubik's experimental result ${ }^{(3)}$ shows that, in some cases of face milling, tool life is kept longer in dry cutting than in wet cutting. All these facts seem to lead us to consider that thermal stress is very responsible for tool rupture. 
In the present work, it is first observed that application of cutting oils of higher cooling efficiency to face milling produces more abrasion at the relief face of cutting edge under certain cutting conditions, and thus, the tool life is shortened. It was next found out through a series of observations on rupture mechanism of tools that tool damages largely depend upon the cracks appearing at rake and relief faces. Also an attempt is made herein to make clear the mechanism of these cracks.

\section{Nomenclatures used}

$\alpha$ : coefficient of thermal expansion

$b:$ width of the material to be cut $\mathrm{mm}$

$\beta$ : relative rate of heat transfer

$c$ : corner angle ${ }^{\circ}$

$d:$ depth of cut $\mathrm{mm}$

$E$ : elastic modulus $\mathrm{kg} / \mathrm{cm}^{2}$

$\varepsilon$ : engage angle ${ }^{\circ}$

$f_{t}:$ feed per tooth $\mathrm{mm}$

$\gamma:$ relief angle ${ }^{\circ}$

$h$ : heat transfer rate cal. $\mathrm{sec}^{-1}{ }^{\circ} \mathrm{C}^{-1} \mathrm{~mm}^{-2}$

$k$ : heat conductivity cal. $\mathrm{sec}^{-1}{ }^{\circ} \mathrm{C}^{-1} \mathrm{~mm}^{-1}$

$L:$ chip-tool interface length $\mathrm{mm}$

$l:$ length of the material to be cut $\mathrm{mm}$

$m$ : constant indicating homogeneity of material used

$\mu:$ Poison ratio

$N$ : number of cracks

$n:$ frequency of cuttings (frequency of impulses)

$R:$ risk of rupture

$P_{V}$ : probability of rupture at the material of volume $\dot{V}$ when loaded with stress $\sigma_{T}$

$P_{V, n}$ : probability of rupture at the material of volume $V$ when loaded with stress $\sigma_{T}$ for $n$ times

$2 r_{m}:$ thickness of test piece $\mathrm{mm}$

$\sigma:$ stress $\mathrm{kg} / \mathrm{cm}^{2}$

$\sigma_{0}:$ strength proper to the test piece $\mathrm{kg} / \mathrm{cm}^{2}$
$\sigma_{T}:$ thermal stress $\mathrm{kg} / \mathrm{cm}^{2}$

$t_{d}:$ temperature difference ${ }^{\circ} \mathrm{C}$

$V:$ volume $\mathrm{mm}^{3}$

$v_{c}$ : cutting speed $\mathrm{m} / \mathrm{min}$

\section{Machines and tools used and materials cut}

Machines: No.3 Vertical milling machine (Cincinatti \& Co.)

Face milling cutter: Bodies of 4 inches in dia. are chiefly used, and those of 10 inches in dia. are used in some parts. Form of cutting edge is; $3^{\circ}$ in rake angle in axial direction, $-10^{\circ}$ in radial direction, $45^{\circ}$ in corner angle, $6^{\circ}$ in relief angle both in peripheral and end cutting edge.

Tool materials: Those used in most parts are of S2 type supplied by a minor maker, and those used in some parts are either S3 or those equivalent to P10, P20, P30 and P40 designated by the ISO system of carbide tool. All the edges are not given tip radius, and the experiments were performed with one blade throughout. Also regrinding was performed by means of “Makino's Universal Tool Grinder", and finishing by a $\$ 220$ diamond wheel of metal bonded.

Materials cut: Carbon steel $(0.4 \% \mathrm{C})$ in a part of the experiment and SCM4 $(b \times l=80 \times$ ca. 500) in the rest of the experiment, both of which were used after tempered and the black scale removed.

\section{Influence of cooling atmosphere on the wear at relief face of tool}

4.1 Difference of relief face wear due to the tool material of different qualities

Effectiveness of lubrication at rake face by the use of cutting fluid is sharply lessened when the cutting speed reaches about $100 \mathrm{~m} / \mathrm{min}$, and is almost lost over the said speed ${ }^{(4)}$. It is also reported ${ }^{(5)}$

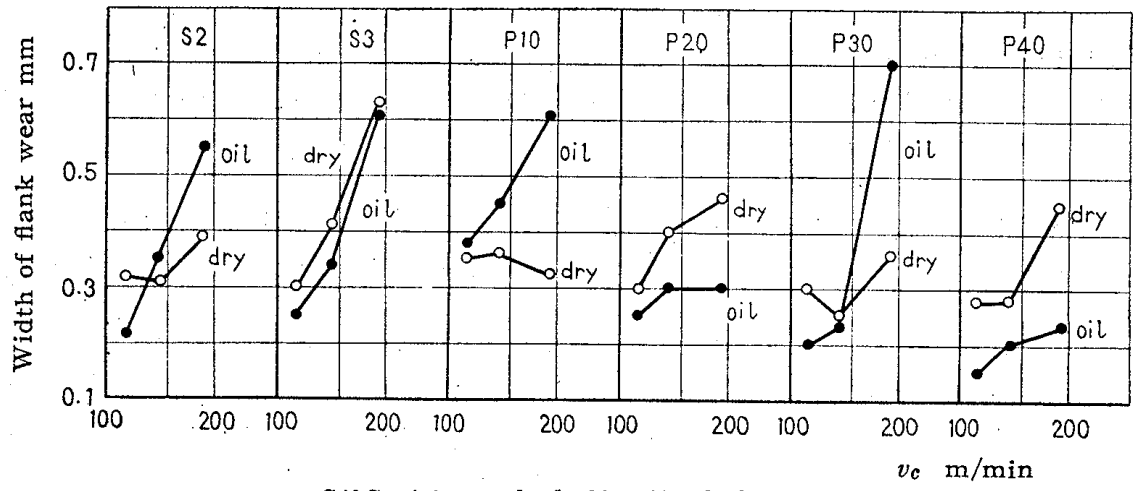

$\mathrm{S} 40 \mathrm{C}, 4$ inch $, b \times l=86 \times 900, d=2, f_{t}=0.12$

Fig. 1 Relations between cutting speed and relief face wear plotted on several tool materials 
that the temperature at rake face differs little either in dry or in wet state if the cutting speed is so high.

In face milling operations, application of cutting fluid helps to cool down the cutting edge at each rotation, and oil films remain sticking all over the rake face until next cutting begins. Such being the advantages of oil application, the author checked on how the cutting condition of either dry or wet state affects the wear of relief face, depending on the tool material, at the speed over $100 \mathrm{~m} / \mathrm{min}$. The checking was performed by measuring the maximum wear width of relief face after a run of a certain length. Cutting fluid used was a sulfurized cutting oil.

In Fig. 1 is shown the change of wear width at relief face of the tool in the case of cutting $\mathrm{S} 40 \mathrm{C}$ of $b \times l=86 \times 450$ with $d=2, f_{t}=0.12$ two times; the curves being plotted against the cutting speed in accordance with the difference of tool material used. Three trends are possibly identified from the figure.

(1) Those which suffer less abrasion if oiled: S3 and others equivalent to P20 or P40.

(2) Those which suffer more abrasion if oiled: Those equivalent to P10.

(3) And the rest which suffer very small abrasion in wet cutting at low cutting speed, and also small abrasion in dry cutting if the speed is raised: $\mathrm{S} 2$ and others equivalent to P30.

It may be admitted as a due criticism that data of this sort should be discussed after mean value is established on the basis of many experimental results. Nevertheless, the result obtained in the above experiment is very suggestive; it may reasonably be presumed from these data that wear mechanism depends not only on toughness to abrasion-resistance of tip at high temperature but also on its thermal conductivity, i. e., those tools considered to have high toughness and thermal conductivity seem, in the experiment, to have good effect of wet cutting, while the others are likely to be made more liable to wearing by the application of oils.

4.2 Difference of relief face wear due to difference in the cooling atmosphere

It may be attributed to thermal stress that application of cutting fluid acts to promote abrasion development on some sorts of tool materials. Then it will also be presumed that increase of temperature difference at the tool surface will result in greater wear, and smaller difference will bring about smaller abrasion. On S2 tip exclusively, therefore, two types of cuttings were tried, one in water to realize the case of wide temperature difference and the other with the blade heated by a gas-burner during its free rotation in the air to prevent quick cooling so as to realize the case of small temperature difference, with the purpose of measuring the maximum wear width of relief face in respective cases.

Results obtained are shown in Fig. 2, where the same data used in Fig. 1 are used again for the cutting with sulfurized cutting oil and that in dry state. The figure reveals a remarkable abrasion on the edge in the case of the cutting in water, and in addition, several cracks running at right angles to the edge were cleary recognizable to the naked eyes in the actual experiment. On the other hand, very small wear was observed in the cutting where the edge was heated during its free rotation in the air. (Temperature of the cutting edge, when heated by the burner, was unknown.) Thus, it is fairly clear from the above result that less abrasion is caused by smaller temperature difference at the edge. But it should be borne in mind that the temperatures at the cutting point are not quite the same for the cutting in the presence of water, that with a cutting fluid, dry cutting and cutting with the blade heated, and so, values of cutting resistance and its direction are different in each case. The above result, therefore, must be regarded as indicating the mixed effects from cutting temperature, cutting resistance and the temperature difference at the cutting edge. In other words, what must be emphasized here is that, if the cooling atmosphere is different, factors other than temperature difference come to act on the development of abrasion at the cutting edge even though the rest of the cutting conditions are kept the same.

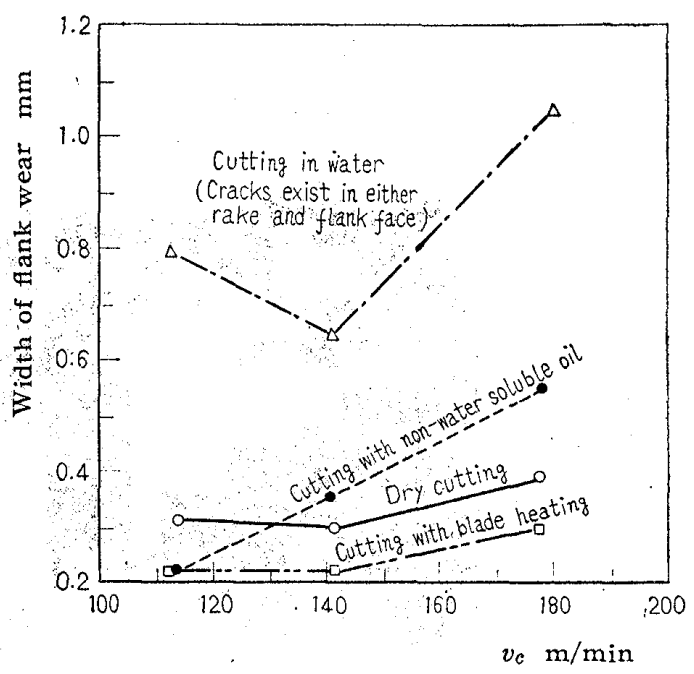

Under the same conditions as in Fig. 1

Fig. 2 Difference in relief face wear due to different cooling atmosphere 
So in Fig. 3, is shown the result of an examination performed to check the state of abrasion by varying the cutter diameter at constant cutting speed and with a fixed engage angle, which means, in macroscopic sense, an experimental condition where temperature difference at the edge alone is varied. Diameters of cutters used were 10 and 4 inches respectively, and tool material was one equivalent to P30. Difference in the diameter gives rise to a small difference in the length of a cut, but is disregarded for the difference being so small. As $b$ in this experiment is kept constant, heating duration is the same in both cases; however, cooling time is to a considerable extent different between them. As clearly observed in the figure, the cutter having 10 inches diameter suffers greater abrasion at any cutting speed than one having 4 inches diameter. The conclusion thus to be drawn from the above experiment is that increase of temperature differernce at the cutting edge results in more abrasion. In this case, however, vibration at the tool due to the difference in diameter should be taken account of also.

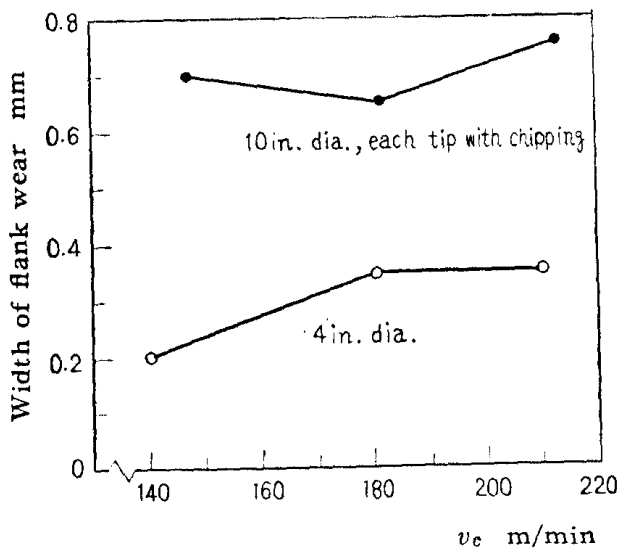

P30, $f_{t}=0.23, \varepsilon=59^{\circ}$

other cutting conditions are the same as Fig. 1

Fig. 3 Difference in relief face wear due to different cutter diameter

\section{Process of wear at cutting edge}

\section{$5 \cdot 1$ Case of greater temperature difference at cutting edge}

Fig. 4 shows the photographs indicating three stages in the wear development at both rake and relief faces of tool, taken in the case of heavy cutting in water at a comparatively high cutting speed. In (a) is observed the state of a tool at the cutting edge when $n=1370$. Here the edge has been a little broken already. Some of the characteristic aspects of this state are that several cracks are seen on the rake face at right angles to the edge, stretching from the edge to the centre portion of the tool; that cracks, though small in number, are seen partially to develop parrallel to the edge; and that cracks are also observed on the relief face in the parallel direction to the edge. (b) of the case of $n=2740$ shows the more progressed state of wear; the cracks are seen on the rake face to have already developed to such an extent, and, in particular, the development of cracks parallel to the edge is remarkable with small portions surrounded by horizontal and vertical cracks breaking away, and thus, a large damage on the relief face takes place. (c) of the case of $n=4110$ presents the worst state of wear, i. e., both relief and rake faces are completely damaged. The information to be drawn from these photos is that two types of cracks appearing either in parallel or at right angles to the cutting edge constitute a major cause of the edge damage.

\subsection{Case of comparatively small temperature} difference at the edge

Cutting in dry state is considered a case where temperature difference at the surface of cutting edge does not vary very much. Fig. 5 is a photograph of damage process of cutting edge in this case. In

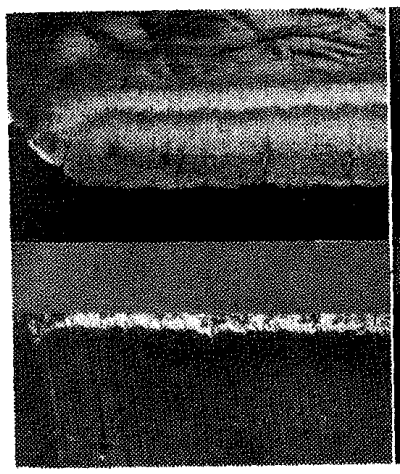

(a) $n=1370$

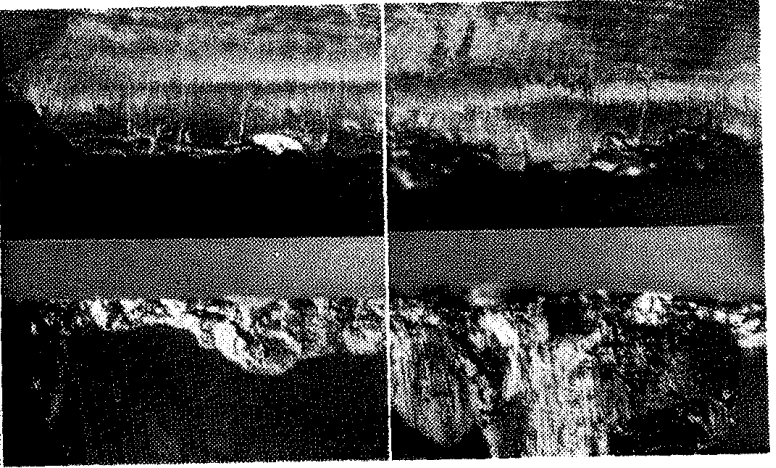

(b) $\mathrm{n}=2740$

$\mathrm{SCM} 4, \mathrm{~S} 2, v_{c}=178, d=3, f_{t}=0.44$ cut in water

(C) $n=4110$

Fig. 4 Advance in wear in case of large temperature amplitude 


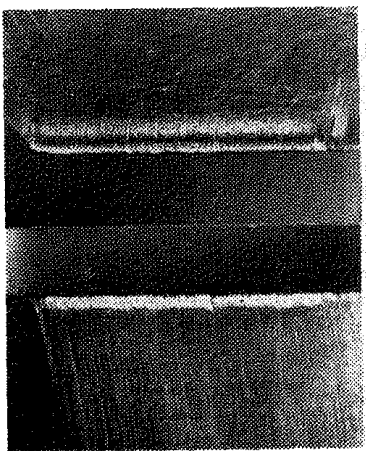

(a) $n=8200$

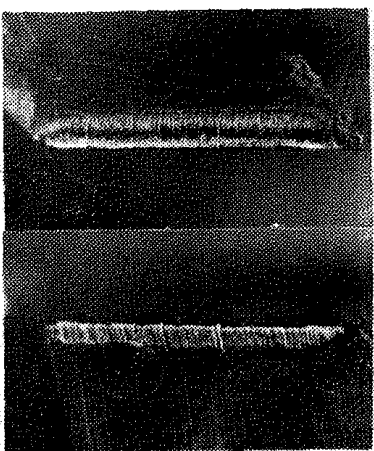

(b) $n=16400$

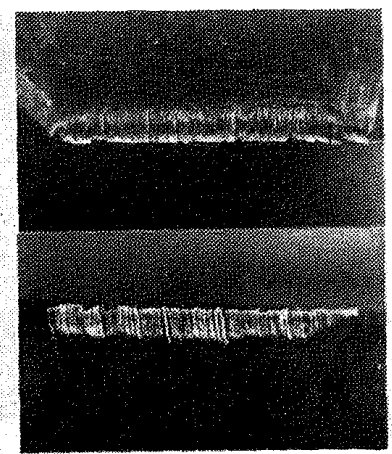

(c) $n=24600$

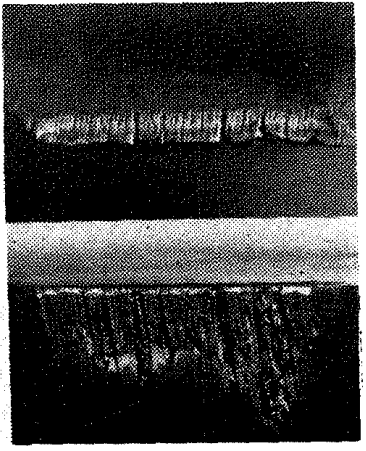

(d) $n=57400$

Fig. 5 Advance in wear in case of comparative small temperature amplitude

(a) are seen, on the rake face, some cracks at almost right angles to the edge, running as long as the chip-tool contact, and in their corresponding position on the relief face, other cracks are observed. When $n$ is increased to the state of (b), fresh cracks are observed on the rake face to appear among the old ones, failing to reach the edge, and another characteristic marking this state is the appearance of parallel cracks in the vicinity of the edge, though difficult to identify clearly on the photo. Two runs of cracks parallel to the edge are recognizable on the relief face also. The wear is further developed in (c), where small abrasion is observed along the parallel cracks on the rake face already seen in (b), and on the relief face, the portion of cracks vertical to the edge is seen to be extremely worn out. The tool is abraded in (d), due probably to further progress of wear at the cracks parallel to the edge which were noticed in (b). The relief face to be seen here is completely worn out along with the cracks vertical to the edge.

Now, as it was verified from the above result that one of the most important factors governing the tool life is the cracks taking place on the rake and relief faces, and it will be of much interest to look into the variety of cutting conditions controlling the occurrence mechanism of the said cracks.

\section{Circumstance for crack appearance}

\subsection{Direction of crack}

Cracks are often observed to appear vertically, say almost at right angles, to the cutting edge. This apparent direction is, if precisely examined, not exactly at right angles to the edge, i. e., it is somewhat inclined. It is also inclined a little to the direction of chip-flow as seen in Fig. 6. The exact direction of the crack development seems to be vertical to that of the diagonal line of chip-tool

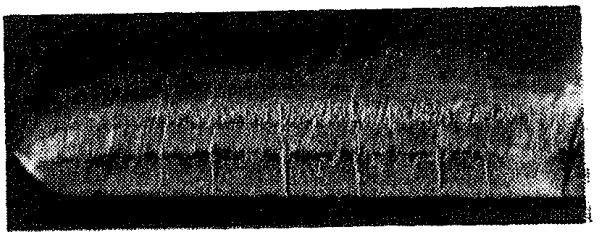

Chip tool interface

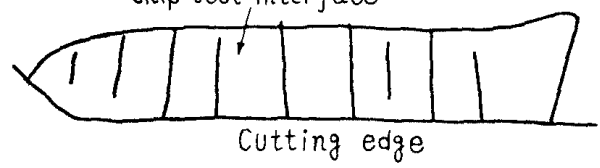

SCM4, S2, $v_{c}=178, d=3, f_{\ell}=0.44$ dry cut (cut in the air)

Fig. 6 Schema of cracks appeared on rake face
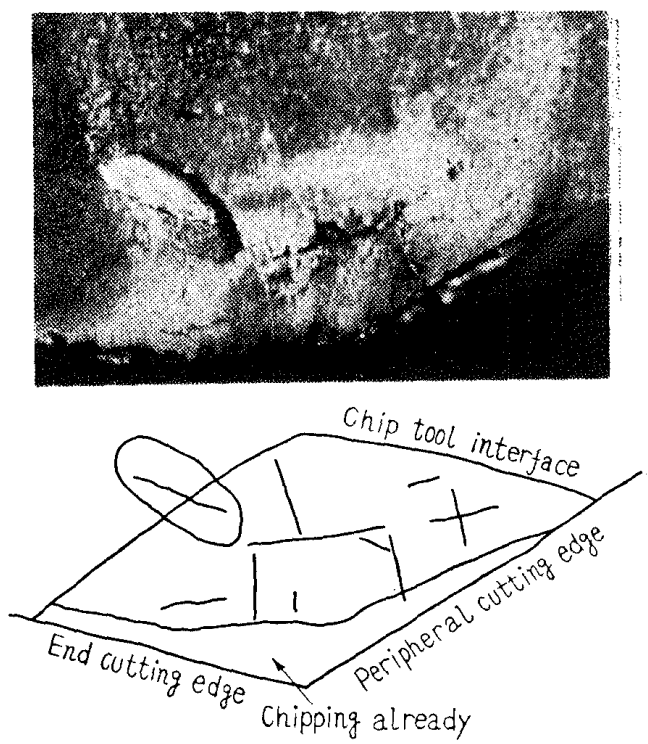

$\mathrm{SCM} 4, \mathrm{~S} 2, v_{c}=141, d=1.5, f_{\iota}=1.38$ cut in water

Fig. 7 Schema of cracks appeared on rake face

interface. The same thing is seen in Okushima: and Hoshi's observation ${ }^{(2)}$.

Fig. 7 illustrates a type of cutting performance in water, where $d$ and $f_{t}$ are adjusted in such a manner as to make the chip-tcol interface almost a rhomb form. Most cracks in this case seem to. 
take place at right angles to the diagonal line of the chip-tool interface. By decreasing $d$ and increasing $f_{t}$, that is, by so arranging as to cut chiefly with the end-cutting edge, you can have cracks running almost vertically to end-cutting edge. Hence it can be said that the direction of crack appearance is, when the chip-tool interface is of rectangular form, at right angles to its longer side, and in the case of a quadrate chip-tool interface, vertical to its diagonal line.

\subsection{Influence of cooling atmosphere}

In Fig. 8 is shown a comparison in the number of cracks to be seen after a definite length is cut between cutting in water and that in the air, where $d$ and $f_{t}$ are kept constant and $v_{c}$ alone is varied. In the case of dry cutting, no crack is seen on the surface of the edge until $v_{c}=76$, and only one crack appears even at the cutting speed beyond the said value as fast as $140 \mathrm{~m} / \mathrm{min}$. On the contrary, in the case of cutting in water it is only until $v_{c}=49$ that no crack is observed, and increase of cutting speed over it results in a rapid increase in the number of cracks to be seen. Hereafter the said cutting speed, at which no crack appears so far, will be named "critical cutting speed" for

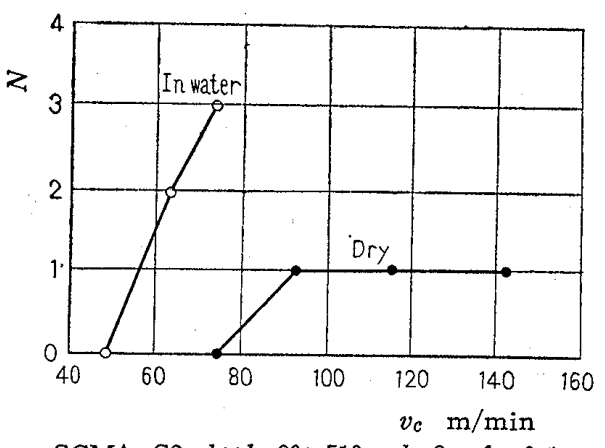

$\mathrm{SCM} 4, \mathrm{~S} 2, b \times l=80 \times 510, d=3, f_{t}=0.1$. $n=5100$

Fig. 8 Difference in generation aspecta of cracks due to different cooling atmosphere

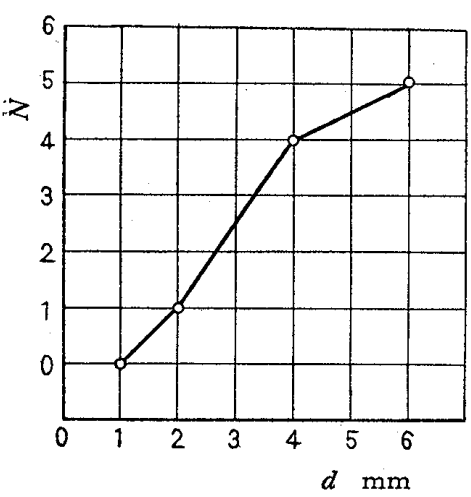

$\mathrm{SCM} 4, b \times l=80 \times 525, \mathrm{~S} 2, v_{c}=141$, $f_{t}=0.33, n=1590$

Fig. 9 Relation between depth of cut and the No. of cracks short. Taking account of the fact that the said critical cutting speed in the cutting in water is largely different from that in the air, and increase in the number of cracks after the first one appears is to a considerable extent different in both cases, we can easily understand that the thermal stress is extremely responsible for crack appearance.

\subsection{Influence of depth of cut}

In Fig. 9 is shown a relation between the depth of cut and the number of cracks to be seen; $v_{c}, f_{t}$ and $n$ were kept constant throughout. $N$ is seen in the figure to increase in a straight line relation to $d$, and thus it is revealed that the length of cutting edge is also an important factor responsible for crack occurrence.

\subsection{Influence of feed}

In Fig. 10, the increase in the number of cracks to appear in response to the variation of feed is plotted, where $v_{c}, d$ and $l$ are kept constant, and $d \gg f_{t}$. In this experiment the cracks are seen to develop at the peripheral cutting edge, and increase their number from 1 at $f_{t}=0.17$ to 5 at $f_{t}=0.26$. But, strange to say, the number shows a decrease if the feed is further increased; 4 at $f_{t}=0.33$ and 3 at $f_{t}=0.42$. This experimental result seems to contradict the afore-mentioned reasoning that cracks come to exist mainly owing to thermal stress, for an increase of feed gives rise to greater thermal stress. But difference in cutting frequencies at each $f_{t}$ ought to be noticed in this case, say 1730 at $f_{t}$ $=0.26,1360$ at $f_{t}=0.33$ and 1070 at $f_{t}=0.42$, a decrease in accordance with the increment of $f_{t}$. What is presumed here is that cracks are possibly coming to exist after the cutting edge is given the thermal stress of a certain value or above, and the number of them depends on the frequency of thermal stress amplitude given to the edge.

In other words, if $n$ had been 1730 at $f_{t}=0.33$ as at $f_{t}=0.26, N$ would have been 5 or more.

Relation between $f_{t}$ and $N$ in the case of $L>d\left(f_{t}>d\right)$ is illustrated in Fig. 11. Cracks observed

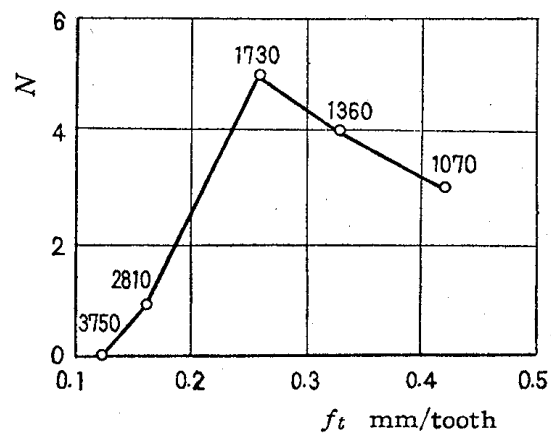

$\mathrm{SCM} 4, b \times l=80 \times 450, \mathrm{~S} 2, v_{c}=141, d=3$, Numerals in the figure mean $n$

Fig. 10 Relation between feed per tooth and the No .of cracks $\left(d \gg f_{t}\right)$ 
herein ran in somewhat vertical direction to the end-cutting edge and $N$ appears to be related with the thermal stress and the frequency at which the cutting edge receives the thermal stress.

\subsection{Influence of cutting speed}

In Fig. 12 are shown some relations between $v_{c}$ and $N$ in the experimental conditions of $d=2, l=$ 450. In the case of $f_{t} \fallingdotseq 0: 1$, the critical cutting speed is $72 \mathrm{~m} / \mathrm{min}$, and even beyond it, we have only one crack to appear. In the case of $f_{t} \fallingdotseq 0.2$, it is $62 \mathrm{~m} / \mathrm{min}$, over which two or three cracks appear. Also in the case of $f_{t} \fallingdotseq 0.4$, the critical cutting speed is much the same as at $f_{t} \fallingdotseq 0.2$; however, increasing the speed over it gives rise to only one or two cracks. Information to be drawn from this figure is that $n$ varies depending on the variation of $f_{t}$. Hence it is further assumed that under the condition of $n=$ const. as mentioned in Fig. 10, the critical cutting speed will be lowered in response to $f_{t}$, thus making $N$ increase. Experimental result obtained here will serve as basic data on which to support the theory that cracks appear probabilistically by thermal stress.

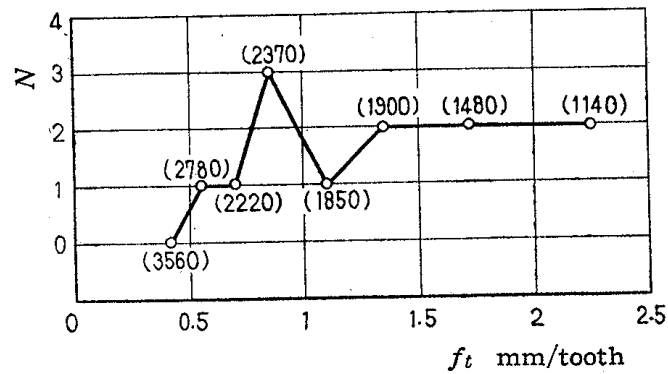

SCM4, $b=80, l:$ var., S2, $v_{c}=141, d=0.5$, Numerals in the figure mean $n$

Fig. 11 Relation between feed per tooth and the No. of cracks $\left(f_{t}>d\right)$

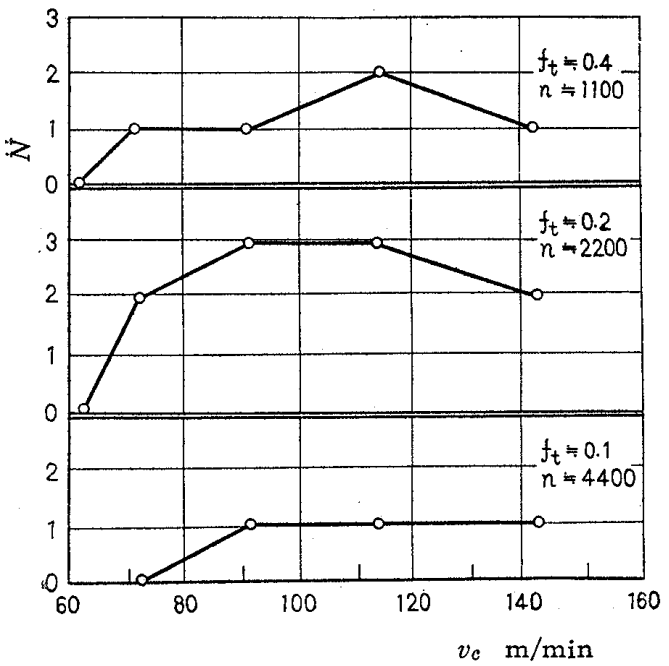

SCM $4, b \times l=80 \times 450, \mathrm{~S} 2, d=2, f_{t} \doteqdot 0.1$, $0.2,0.4$

Fig. 12 Relation of the No. of cracks against cutting speed
As far as the author's experiment is concerned, the effect of cutting speed shows a saturation curve in accordance with the increment of cutting speed as illustrated with three curves in the figure.

\subsection{Influence of width of the material cut}

As variation of $b$ results in different ratios between the cutting time and the free rotation time though all of the other cutting conditions are the same, it will reasonably be presumed that the difference of temperature at the cutting edge, i.e., the thermal stress therein will be changed eventually. The critical feed per tooth is checked in Fig. 13, where $b$ alone is varied. The fact that, while the critical feed at $b=80$ is $0.12 \mathrm{~mm}$, it is $0.048 \mathrm{~mm}$ at $b=60$ may be attributed to the large temperature difference at the edge in the latter case. And the fact that the critical feed is faster when $b=20$ or 40 than when $b=60$ will be also explained by small temperature difference at the edge though cooling time may be long because of short heating duration. As $n$ was not constant in this experiment, the comparison illustrated here is not made under the same conditions. If the critical feed had been measured under the same $n$, more definite difference would have been obtained.

In short, cracks are not likely to appear when the width of the material to be cut is much the same as the cutter diameter, and as the width gets smaller, cracks appear more easily. And no cracks appear

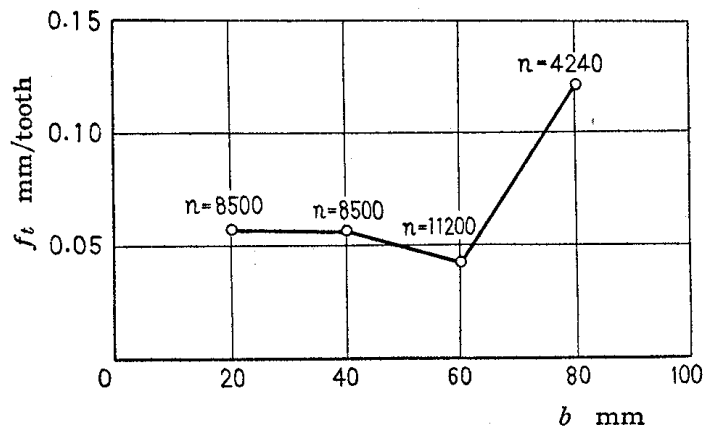

SCM $4, l=510, v_{c}=141, d=3, \varepsilon=53^{\circ}$

Fig. 13 Relation between width of work and critical feed

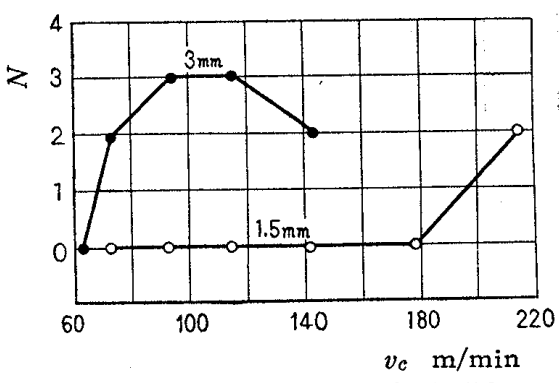

SCM4, S2, $d=2, f_{t}=0.2, l=450$, thickness of tip $3 \mathrm{~mm}, 1.5 \mathrm{~mm}, n=2200$

Fig. 14 Relation between thickness of tool ti $p$ and the No. of cracks 
easily when the width is further smaller.

6.7 Influence of the thickness of carbide tip

In general the rupture due to thermal stress is strongly dependent on the thickness of the specimen. So, in Fig. 14 are shown the results of observations made concerning the states of crack appearances on the materials with a thickness of $1.5 \mathrm{~mm}$ and 3 $\mathrm{mm}$ respectively under the same conditions.

In the material $3 \mathrm{~mm}$ in thickness, cracks start to appear at $v_{c}=72$, while in the material $1.5 \mathrm{~mm}$ in thickness, they do not come to exist until $v_{c}=178$, and are clearly observed at $v_{c}=212$.

Thus it is verified from the above result that the thickness of tip affects strongly the crack appearance. Now it is understood that since cracks are much related to the thickness of tip as much as the size of heat source (chip-tool interface) as already shown in Figs. 9 and 10, they have certain size effects.

\subsection{Process of crack appearance}

Now, whether cracks come to exist all at once or gradually presents an interesting problem. As presumed in Figs 10, 11 and 12, cracks have much to do with the thermal stress, while at the same time, they are strongly controlled by the stress frequency. Accordingly, by the use of different tips, measurements of the number of cracks to be observed were performed at intervals through one cutting experiment, such as at the intervals of $50 \mathrm{~mm}, 100$ $\mathrm{mm}, 250 \mathrm{~mm}$ and $500 \mathrm{~mm}$ in cutting length.

In Fig. 15, where the cutting length and the cutting frequency are taken as ordinate, $N$ of the four specimens used seem to be almost fixed against certain values of $\dot{n}$ or $l$, each tracing somewhat saturation curves, that is, many of the cracks appear while $n$ is small, but not appearing all at once ; they are observed to increase in the form of a saturation curve.

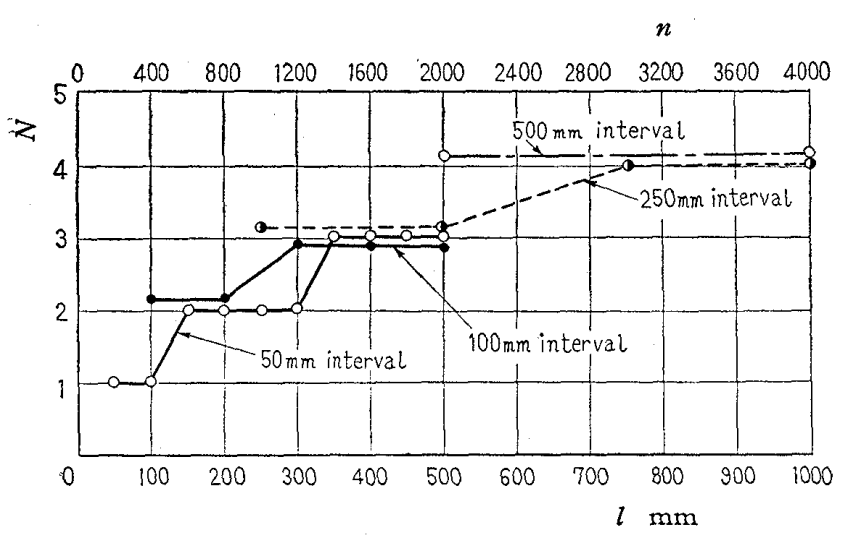

$\mathrm{SCM} 4, b=80, \mathrm{~S} 2, v_{c}=141, d=3, f_{t}=0.25$, intervals of measuring $50 \mathrm{~mm}, 100 \mathrm{~mm}$, $250 \mathrm{~mm}, 500 \mathrm{~mm}$

Fig. 15 Process of crack generation

\section{Mechanism of crack appearance}

In the previous chapter are described the circumstances and conditions for crack appearance, and the characteristic conclusions to be drawn from the results are as follows;

(a) Cracks are likely to appear in the case of an effective tool-cooling atmosphere or under severe cutting conditions, i.e., the thermal stress arising from great temperature difference plays an important role in crack appearance.

(b) Size of heat source and thickness of tip are much responsible also, i.e., crack appearance depends on the specimen's size.

(c) Cracks do not come to exist all at once ; probability for crack appearance due to one thermal stress is quite small.

\subsection{Thermal stress given to the surface of} the tool

First, in consideration of (a), it is reported ${ }^{(6)}$ from the standpoint of thermal shock study that, when an infinite sheet of $2 r_{m}$ in thickness is heated or cooled uniformly from both sides, the thermal stress on the surface is expressed as ;

$$
\sigma_{T}=A \frac{E \cdot \alpha \cdot \beta \cdot t_{d}}{1-\mu}
$$

provided $\beta=r_{m} h / k$ and $A=$ const.

The above equation is not applicable to the present study because the tool under investigation is neither an infinite sheet nor is heated and cooled likewise from both sides. But it will as well be applicable for estimating the thermal stress on the surface of a tool without serious errors, if a new constant $A^{\prime}$, which serves as form-coefficient too, is substituted for the constant $A$ in the equation.

$$
\sigma_{T}=A^{\prime} \frac{E \cdot \alpha \cdot \beta \cdot t_{d}}{1-\mu}
$$

It is clear from Eq. (1) that the thermal stress on the tool face is governed by $r_{m}, h$ and $t_{d}$ the tool material is fixed: The larger the values of the said factors, the larger $\sigma_{T}$ becomes, thus resulting in damage on the surface, that is, the appearance of a crack.

Under heating $\sigma_{T}$ means compressive stress, and under cooling, tensile stress, and the stress amplitude between compressive and tensile state is generally believed to contribute strongly to giving rise to fatigue fracture on ductile materials, but in the case of brittle materials, the tensile stress is considered to be chiefly responsible for the rupture : the fact that compressive strengh of brittle materials is several times as much as their tensile strength may support the above consideration. Thus, cracks must be considered to appear due to the tensile thermal 
stress in cooling process, provided that the tensile stress $\sigma_{T}$ exceeds the tensile strength of tool material.

Then, if the value of $\sigma_{T}$ is larger than the breaking strength of the tool, cracks ought to appear after the surface undergoes one thermal stress, that is, after one cut. Also, if $\sigma_{T}$ is smaller than the breaking strenth of the tool, however frequently the thermal stress may be applied, no crack is due to appear.

\subsection{Mechanism of crack appearance}

Since it was verified that the maximum stress theory is incapable of explaining the experimental facts observed in the present study, an attempt was next made to adopt the Weibull's theory ${ }^{(7)}$, that is, if inhomogeneity of the material is taken into consideration, rupture comes to take place on the material with a certain probability dependent on the material volume, the stress and the stress distribution. This Weibull's theory is generally considered to be more suitable to the cases where brittle materials are used.

The fundamental Eq. of Weibull's theory is as follows :

$$
R=\frac{1}{\sigma_{0}{ }^{m}} \int_{V} \sigma^{m} d V
$$

Application of the Weibull's theory to the explanation of the mechanism of crack appearance will be described hereafter. Since it was already observed that cracks take place only in the chip-tool interface, the tool tip is here assumed as a test piece of $2 r_{m}$ in thickness used in a bending test where the length of chip-tool contact is $L$ and that of cutting edge $d \cdot \sec c$, as illustrated in Fig. 16. By
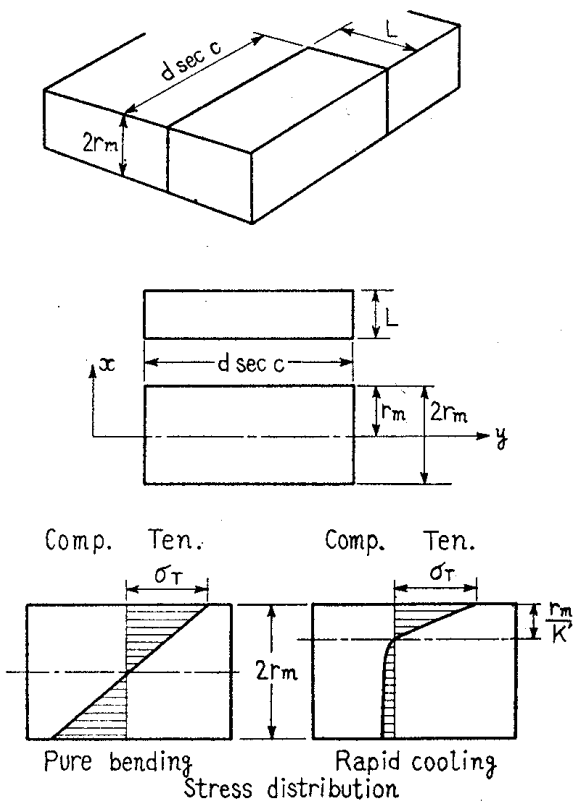

Fig. 16 being cooled, the tip is given a tensile stress in the upper part over $y$-axis and a compressive stress in the lower part, thus yielding cracks on its surface owing to thermal stress $\sigma_{T}$. (The test being considered as pure bending test, the specimen has $y$-axis in the center of its thickness.)

The stress at the point apart from $y$-axis by the distance $x$ is expressed as $x \sigma_{T} / r_{m}$, and the volume receiving this stress $L d \sec c d x$. Substituting the above values into Eq. (2) and integrating it, we have

$$
R=\frac{r_{m} L \cdot d \sec c}{m+1}\left(\frac{\sigma_{T}}{\sigma_{0}}\right)^{m}
$$

or

$$
R=\frac{V}{2(m+1)}\left(\frac{\sigma_{T}}{\sigma_{0}}\right)^{m}
$$

The above equations are formulated on the basis of simple bending, that is, the $y$-axis runs just in the center of the thickness, but when the tip is quickly cooled, the $y$-axis moves upward as shown in Fig. 16 , and the curve of tensile stress can be aproximated as a straight line. Therefore, we have

$$
R=\frac{V}{2 K(m+1)}\left(\frac{\sigma_{T}}{\sigma_{0}}\right)^{m}
$$

provided that $K$ is a plus constant over 1 , increasing in response to $\beta$. Probability of rupture $R$ is 'defined as

$$
\begin{aligned}
& R=-\log \left(1-P_{V}\right), \text { we have } \\
& -\log \left(1-P_{V}\right)=\frac{V}{2 K(m+1)}\left(\frac{\sigma_{T}}{\sigma_{0}}\right)^{m} \\
& P_{V}=1-\exp \left[-\frac{V}{2 K(m+1)}\left(\frac{\sigma_{T}}{\sigma_{0}}\right)^{m}\right]
\end{aligned}
$$

or

$$
P_{V}=1-\exp \left[-\frac{r_{m} L \cdot d \sec c}{K(m+1)}\left(\frac{\sigma_{T}}{\sigma_{0}}\right)^{m}\right]
$$

where $P_{V}$ means the probability that a test piece of volume $V$ is ruptured under the stress level of $\sigma_{T}$, showing such trend as illustrated with a solid line in Fig. 17. Also $P_{V}$ is dependent on $\left\{r_{m} L d \sec c \mid\right.$ $K(m+1)\}\left(\sigma_{T} / \sigma_{0}\right)^{m}$. If the test piece is completely homogeneous, $m \rightarrow \infty$, and $P_{V}$ has the trend shown by a dotted line in the figure agreeing well with the explanation by the maximum stress theory. Eqs. (6)

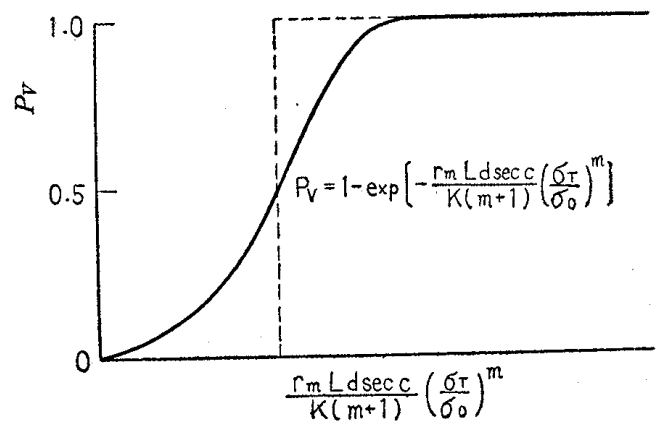

Fig. 17 Curves of probability of crack appearance 
and (7) indicate the probability of rupture when the thermal stress is applied only once. So, if the surface experiences thermal stresses repeatedly with the frequency of $n$, the probability of rupture $P_{V n}$, will be expressed as

$$
P_{V},{ }_{n}=n \cdot P_{V}
$$

\section{7•3 Consideration}

The $\sigma_{T}$ expressed in Eq. (1) and $P_{V}$ in Eq. (7) can account for the entire mechanism of crack appearance. First, its characteristic dependency on thermal stress will be explained as follows : According to Eq. (1), $\sigma_{T}$ is to increase proportionally to the thickness of tip, heat transfer rate of the surrounding atmosphere and the temperature difference at the surface of tool respectively, then, if $\sigma_{T}$ is increased, $P_{V}$ approaches 1 in respect to $\sigma_{T}{ }^{m}$ according to Eq. (7). Thus, the dependency of cracks on thermal stress is explained, that is, the results obtained in Figs. 8, 10, 12 and 13. The fact that power of Eq. (7) is multiplied by $1 / K$ in the case of quick cooling, and thus $P_{V}$ is calculated much smaller than in the case of slow cooling will not matter very much in practical sense, for the said fact comes to exist only when the surface stress is equal in both coolings, and in actuality, it differs to a great extent between them.

Next, the problem of size effect will be easily explained since the term of volume is included by the power of $P_{V}$. Influences of depth of cut (Fig. 9), feed (Fig. 11) and thickness of tip (Fig. 14) are considered to come from an increment of $P_{V}$ due to the increase in volume. Another experimental result that the direction of cracks lies at right angles to the longer side of the chip-tool interface when it is rectangular, or at right angles to the diagonal line when the chip-tool interface is of rhomb form will be interpreted as follows : in Fig. 18 are considered a chip of $2 r_{m}$ in thickness and a heat source of rectangular surface whose two sides are $x$ and $y$ respectively $(y>x)$. The volume of a minimum slice obtained by an arbitrary cutting parallel to the thickness direction takes the maximum value when
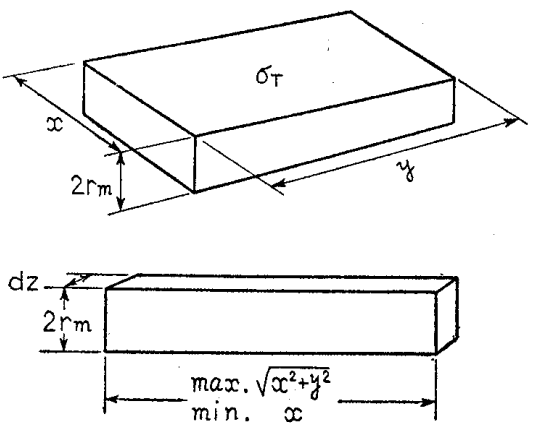

Fig. 18 it is cut in the direction of the diagonal line of the surface, the value being $2 r_{m} \sqrt{x^{2}+y^{2}} d z$ (where $d z$ indicates the minute thickness). Taking this minute volume into consideration and provided that cracks will appear most probably in the direction of a diagonal line. The trends observed in Figs. 6 and 7 are agreeable in this respect. And in practical work, the depth of cut is far larger than the feed, i.e., $y \gg x$, rendering $\sqrt{x^{2}+y^{2}} \fallingdotseq y$ and thus cracks are seen to develop vertically to the main cutting edge.

Another explanation for the saturation-curve-like trend of crack appearance is this ; Eq. (6) will be written as $P_{V}=1-e^{-\kappa V} \quad(\kappa:$ const.) provided that the cutting conditions are kept the same throughout the operation. Appearance of cracks in the number of $N$ divides the edge into the blocks of the number of $(N+1)$. The mean volume of one block becomes $V /(N+1)$. Therefore, $P_{V}$ is expressed as $\left(1-e^{-\kappa V /}\right.$ $\left(N^{+1)}\right)$, and $P_{V}$ becomes smaller in proportion to an increase of $N$. Thus cracks have to increase in the form of a saturation curve.

Next, another result obtained in Fig. 4 that cracks show their first appearances vertically to the edge and then parallel to it will be explained; $y>x$ in the original state is changed into $x>(y / N+1)$ after the appearances of cracks in the number of $N$, and thus cracks have no choice but to appear vertical to the side of $x$. Another fact that, on the rake face, though cracks are likely to appear in large number in the direction vertical to the edge, they are hardly observed in the direction of diagonal lines of the small blocks nor parallel to the edge is considered to be attributed to the influence of the free end or the un-evenness of temperature difference within the chip-tool interface, but future study is needed for the complete clarification.

Some of the observations in Figs. 10 and 12 that $N$ is small regardless of large value of $f_{t}$ may be due to the small value of $n$ calculated by Eq. (8).

\subsection{Protection against cracks}

All of the above considerations leads us to the conclusion that such a cutting condition as to lessen the value of $P_{V}$ in Eq. (7) works effectively to minimize the crack appearance. In the actual sense, it means a decrease in the values of $2 r_{m}, L, d \sec c$ and $\sigma_{T}$ at the same time, an increase in $\sigma_{0}$.

A) As ideal cutting conditions, the following are recommended :

a) To minimize the temperature difference at the cutting edge.

b) To lower the maximum temperature as much as posible.

c) And to minimize the chip-tool interface as well as to make it a quadrant form, and 
also thinner tips are recommended.

B) As an ideal material of the tool :

a) Those of high rupture strength $\left(\sigma_{0}\right)$.

b) Those of smaller elastic modulus, coefficient of thermal expansion and Poisson ratio.

c) And those of high heat conductivity. It should be emphasized here that the above characteristics indicating the resistance of a material to rupturing have still nothing to do with abrasion-resistance at high temperature.

\section{Conclusion}

In respect to cracks causing large damage to face milling tool, it was contended that they appear with a certain probability depending on the thermal stress, the size of heat source or the thickness of tool material, and the results obtained in the experiment showed good accordance with theory.

The author expresses his hearty acknowledgement to Prof. M. Okoshi who gave helpful advices in the course of this investigation. Also he would like to thank Mr. Mitsuharu Tago for his cooperation.

\section{References}

(1) H. Opitz u. Frölich : VDI-Z, Bd. 96 (1954), S. 822.

(2) Okushima and Hoshi: Trans. Japan Soc. Mech. Engrs., Vol. 27, No. 178 (1961), p. 843; Preprint of the 39 th the National Lecture Meeting, No. 58 (Nov. 1961), p. 61 .

(3) J.R. Roubik: Lubric. Engng., Vol. 8 (1952), p. 235.

(4) M.C. Shaw and P.A. Smith: Tool Engng., Vol, 36 (1956), p. 111.

(5) Shōnozaki and Hiyoshi : Lubrication, Vol. 1, No. 3 (1956), p. 159 .

(6) W.D. Kingery: Jour. Amer. Chem. Soc., Vol, 38 (1955), p. 3.

(7) W. Weibull: Ing. Vetenshapsakademiens, Nr. 151 (1939), S. 45; Physik. Ber., Bd. 20 (1939), S. 1668.

\section{Discussion}

T. Hosm : (1) Damages to the cutting edge are mainly caused by cracks nearly perpendicular to the cutting edge and ones nearly parallel to it, as the author has revealed. Particularly the latter kind of cracks is deemed more important as the direct factor causing damage to the cutting edge. Meanwhile the picture in Fig. 4 shows how cracks of the latter kind occur very close to the cutting edge. In the area directly adjoining the cutting edge, which represents a free end, the thermal stress in the perpendicular direction to the cutting edge is presumably small. Is it right to assume that the latter kind of cracks occurring very close to the cutting edge is also attributed to the thermal stress?

(2) The author states from observation of the picture in Fig. 6 that the cracking direction is at right angles to the diagonal direction of chip contact. But the picture shows rather that cracks are nearly perpendicular to the cutting edge and slightly inclined near the face cutting edge under the influence of the latter.

Or in Fig. 7 the actual cutting edge looks like an arc on account of damage; the chip contact area emerging white in the picture assumes not a rhombic form but a strip form following the arc-like cutting edge and the longitudinal and transverse cracks might more appropriately be regarded as running nearly at right angles or parallel to the cutting edge rather than at right angles to the diagonal line of chip contact area.

Meanwhile it would be impossible to deduce that the cracking direction would be perpendicular to the diagonal line from the fact that there is the highest probability for cracks to originate from the microvolume in the diagonal direction. Thus, the discussor thinks that a principal stress occurs in the direction along the cutting edge and the cracks develop perpendicular to the stress, i.e., to the cutting edge ; meanwhile, another stress occurs in the other direction, that is, perpendicular to the cutting edge and if this stress is large, cracks also occur in the parallel direction along the cutting edge.

\section{Author's closure}

(1) It is seen from Fig. 4 (a) that the cracks in parallel with the cutting edge direction develop in a zone apart from the edge by one third of toolchip contact length. Cracks seen in (b) appear close to the edge, but this is only due to breakage of cutting edge (as is clearly shown in photos of relief face) and the cracks themselves are all the same as those to be seen in (a).

As you have pointed out, it seems likely that parallel cracks are rather difficult to take place in the vicinity of the edge, except in the case when the width of a worn out portion on the relief face becomes wide enough to generate excessive heat, for it is very probable that this heat may possibly exercise some effect on the stress which acts on the rake face.

(2) No one may be able to conclude positively from Fig. 6 that cracks to be observed in the photo run in a direction exactly at right angles with the cutting edge. Also close investigation on the worn out edge shown in Fig. 7 after restoring 


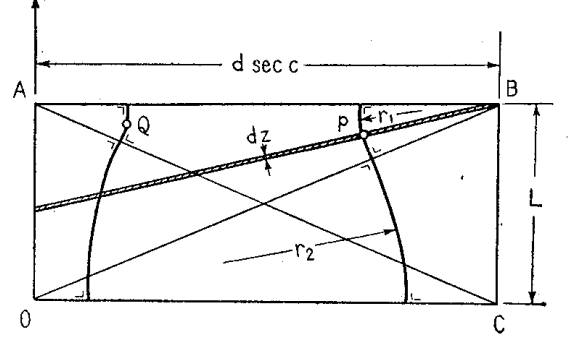

Append-Fig. 1

its abraded parts will reveal that far more cracks are observable at right angles to the pentagonal line than in any other directions.

Nevertheless, the above description will not be sufficient to give the conclusion that cracks appear in a direction at right angles to the diagonal line, and therefore, supplementary reasonings will be given below.

In the Append-Fig. 1, OABC is taken as chip-tool contact area, $\mathrm{OA}$ as the end edge, and $\mathrm{OC}$ as peripheral edge. By assuming that there exists a defect at a point $P$ located in the right half of the area and cracks appear through the point $P$, if the tensile thermal stress at $P$ is uniform in every direction, we will find the highest probability of crack development along the line which falls at right angles to BP direction (minimum width $d z$ ) from Eq. (17).
In case when stresses are given through shocks, a crack continues to develop with inertia, but in case when thermal stresses are given in comparatively modest manner as in dry-cutting and its development is quasi-static, a trace of crack run will draw an arc of a circle around the center $B$ with radius of $\mathrm{BP}=\boldsymbol{r}_{1}$, and after crossing with the diagonal line BO, will also draw an arc of a circle but around 0 this time and the radius will be $r_{2}$. If a defect is: found at a point $Q$ located in the left half of chiptool contact area, a symmetrical trace of crack occurrence will be found in the same manner as. above.

The explanation given above, however, concerns only the typical case of crack occurrence. The photograph shown in Fig. 6 seems to be in fairly good agreement with the above explanation.

$W i t h$ respect to the direction of a main stress, the author holds the view point that it is uniform in every direction rather than that it takes two directions. It is of course a matter of fact that there is a difference in temperature distribution on the surface of cutting edge and so the thermal stress is, strictly speaking, not uniform in all directions, but it will also be practical to consider that. the difference is small enough to be disregarded except for the limited area near the edge.

Errata (Vol. 5, No. 19, August, 1962)

\begin{tabular}{|c|c|c|c|c|c|}
\hline Author & Page & Column & Line & For & Read \\
\hline I. YAMAKAWA & 399 & right & Fig. 10 & by the displacement & by the force \\
\hline H. Miyashiro & 400 & left & Fig. 11 & by the force & by the displacement \\
\hline \multirow{7}{*}{$\begin{array}{l}\text { T. SAto, I. Michiyoshi, } \\
\text { and R. Matsumoto }\end{array}$} & 484 & left & Fig. 5 & $h, \cdots \cdots n_{A}, u_{B} \%$ & $h, \cdots \cdots n_{A}, n_{B} \%$ \\
\hline & 507 & right & 13 & $C_{2}=\cdots \cdots c_{p a} \frac{P}{R} V_{a} S$ & $C_{2}=\cdots \cdots c_{p a} \frac{P}{R} V_{a} S_{0}$ \\
\hline & 507 & right & Eq. (14) & $+\boldsymbol{E}(D) \frac{\partial \boldsymbol{E}}{\partial \boldsymbol{D}}=$ & $+\boldsymbol{E}(D) \frac{\partial \boldsymbol{F}(D)}{\partial \boldsymbol{D}}$ \\
\hline & 508 & left & 16 & 1 & 1 \\
\hline & & & & $b V_{f}$ & $b V_{f}$ \\
\hline & 508 & right & 7 & $\boldsymbol{F}(D)=\frac{V_{f_{0}} S}{V_{f} S} l$ & $\boldsymbol{F}(D)=\frac{V_{f 0} S_{0}}{V_{f} S} l$ \\
\hline & 508 & right & 23 & where $\boldsymbol{F}_{w}(D)$ is & where $\boldsymbol{F}_{w}(D)_{0}$ is \\
\hline
\end{tabular}

